\title{
Impacto das Ondas de Calor no Conforto Térmico Humano na Região da Floresta Nacional do Tapajós, Oeste do Pará
}

\author{
Tiago Bentes Mandú ${ }^{1}$, Ana Lucia da Silva Nascimento ${ }^{1}$, William Duarte Jacondino ${ }^{1}$ \& Ana Carla dos Santos Gomes ${ }^{2}$
}

Recebido em 15/09/2020 - Aceito em 03/05/2021

1 Instituto Nacional de Pesquisas Espaciais. Brasil.<tiagobentes1@gmail.com, analuciasne@gmail.com, williamjacondinoufpel@gmail.com>.

2 Universidade Federal do Oeste do Pará/UFOPA. Brasil. <anacarlasg02@gmail.com>.

\begin{abstract}
RESUMO - Ondas de calor têm sido mais recorrentes mediante um cenário de aquecimento global. As temperaturas extremas resultam no desconforto térmico humano por calor, o que pode causar e potencializar comorbidades e levar ao óbito em casos extremos. Avaliou-se neste estudo a ocorrência de ondas de calor e sua relação com o desconforto térmico humano na região da Floresta Nacional do Tapajós, localizada no estado do Pará, Brasil. Os dados foram obtidos a partir da estação meteorológica convencional do Instituto Nacional de Meteorologia, durante o período de 1971 a 2019. O conforto térmico humano foi estimado utilizando o Índice de Desconforto de Thom, que é uma metodologia empírica capaz de representar o ambiente térmico por meio de dados de temperatura máxima e umidade relativa do ar, enquanto que as ondas de calor foram identificadas a partir do índice CTX90pct. Os resultados indicam que há um aumento no desconforto térmico humano; com maiores índices de desconforto na estação menos chuvosa e potencializadas em períodos de seca na região. O Índice de Desconforto mostrou que, na presença de ondas de calor, o desconforto térmico sentido pela população aumenta em cerca de $40 \%$. Os resultados também evidenciam que as ondas de calor potencializam os níveis de desconforto, que podem causar efeitos na saúde pública e atividades socioeconômicas da região.
\end{abstract}

Palavras-chave: Amazônia; unidades de conservação; estresse térmico; eventos climáticos extremos; sensação térmica.

\section{Impact of Heat Waves on Human Thermal Comfort in the Tapajós National Forest, West of Pará}

\begin{abstract}
Heat waves have been more recurrent through a global warming scenario. Extreme temperatures have some rate of human thermal discomfort due to heat, which can cause and potentiate comorbidities and lead to death in extreme cases. This study evaluated the occurrence of heat waves and their relationship with human thermal discomfort in the Tapajós National Forest region, located in the state of Pará, Brazil. Data were obtained from the conventional weather station of the National Institute of Meteorology, during the period from 1971 to 2019. Human thermal comfort was estimated from the Thom Discomfort Index (DI), which is an empirical methodology capable of representing the thermal environment by means of data of maximum temperature and relative humidity and heat waves were estimated using the CTX90pct index. with higher rates of discomfort in the less rainy season and potentiated in periods of drought in the region. The DI showed that in the presence of heat waves, the discomfort caused by heat felt by the population increases by about $40 \%$. The results presented added to the increase in discomfort can cause effects on public health and socioeconomic activities in the region.
\end{abstract}

Keywords: Amazon; conservation units; thermal stress; extreme weather events; thermal sensation.

\section{Impacto de las Olas de Calor en el Confort Térmico Humano en la Región del Bosque Nacional Tapajós, al Oeste de Pará}

RESUMEN - Las olas de calor han sido más recurrentes en un escenario de calentamiento global. Las temperaturas extremas resultan en malestar térmico humano debido al calor, térmico humano debido al calor, que puede causar y potenciar comorbilidades y conducir a la muerte en casos 
extremos. Este estudio evaluó la ocurrencia de olas de calor y su relación como malestar térmico humano en la región del Bosque Nacional Tapajós, ubicada en el estado de Pará, Brasil. Los datos se obtuvieron de la estación meteorológica convencional del Instituto Nacional de Meteorología, desde 1971 hasta 2019. El confort térmico humano se estimó mediante el índice de malestar de Thom, que es una metodología empírica capaz de representar el ambiente térmico mediante datos de temperatura máxima y humedad relativa, mientras que las olas de calor se identificaron a partir del índice CTX90pct. Los resultados indican que hubo un aumento en el malestar térmico humano; Con mayores niveles de malestar en la estación menos húmeda y aumentado durante los períodos secos en la región. El Índice de Malestar mostró que la presencia de olas de calor, o malestar térmico que siente la población, aumenta aproximadamente en $40 \%$. Los resultados también muestran que los sofocos aumentan los niveles de malestar que pueden causar efectos en la salud pública y las actividades socioeconómicas de la región.

Palabras clave: Amazônia; unidades de conservación; estrés termal; eventos climáticos extremos; sensación térmica.

\section{Introdução}

Eventos climáticos extremos têm sido recorrentes mediante o cenário de mudanças climáticas, trazendo consigo vários impactos negativos à biodiversidade e a sociedade, como por exemplo o aumento da temperatura média global, mudança no regime de precipitação $e$ mudanças no padrão de distribuição das espécies. (Stott et al., 2013; Silva, 2018; Kong et al., 2020). Neste cenário estão as Ondas de Calor (OC), que segundo a literatura tem amplas definições, entretanto, todas convergem para um período prolongado com temperaturas extremas para uma determinada região (Frich et al., 2002; Bitencourt, et al., 2016; Geirinhas et al., 2017; Shiva et al., 2019; Mandú et al., 2020b).

$\mathrm{Na}$ literatura são encontrados inúmeros estudos que indicam o aumento da temperatura média global para cenários futuros (Marengo \& Valverde, 2007; Artaxo, 2014; Marengo, 2014; Mitchell et al., 2016), o que favorece o aumento na frequência de ocorrência de extremos climáticos, como as OCs. No Brasil, Bitencourt et al. (2016, 2020) mostraram que o número de casos de OC apresentaram acréscimo nas últimas cinco décadas; fato observado também para todo o cone sul da América do Sul, que atingem recordes de temperaturas mediante o cenário global (Reis et al., 2017; Jacondino et al., 2018).

A ocorrência de OC podem apresentar fatores de risco para morbidade e mortalidade relacionadas ao desconforto térmico, sendo os principais afetados idosos, crianças, doentes/ acamados, regióes com alta densidade populacional, baixa renda, pouca cobertura vegetativa ou regiões protegidas que vem sendo degradadas
(McGeehin \& Mirabelli, 2001; Jenerrete et al., 2007; Geirinhas et al., 2020). Tais impactos em áreas metropolitanas podem ocasionar e intensificar a formação de ilhas de calor que podem aumentar o estresse térmico e pode em situações extremas levar a óbito (Laaid et al., 2012; Mitchell et al., 2016). Os impactos das temperaturas extremas são observados também no aumento dos riscos de incêndios florestais, na produtividade agrícola, agropecuária e na demanda por consumo de energia elétrica e hídrica (Rusticucci \& Barrucand, 2002; Lima et al., 2013; Bitencourt et al., 2016).

Estudos recentes buscam entender a influência das variáveis meteorológicas com o desconforto humano, através de índices que buscam relacionar condições de estresse térmico (dentre eles por calor) a comorbidades e doenças relacionados à saúde humana (Nastos \& Matzarakis, 2006; Ashrae, 2009; Parsons, 2014; Nick \& Nedel, 2018). Por exemplo, um estudo conduzido por Geirinhas et al. (2020) detectou um aumento na mortalidade da população relacionado à presença de $\mathrm{OC}$ extremas durante o verão na região metropolitana do Rio de Janeiro, destacando uma maior mortalidade relacionada a períodos consecutivos por dias com extremo calor, que por dias esporádicos.

Há uma ampla gama de estudos na literatura que buscam compreender o impacto das OCs em grandes cidades ou regiões populosas. Porém, em regiões interiorizadas essa demanda ainda é incipiente. Devido ao processo de urbanização $e$ degradação próximo a áreas preservadas, vê-se a grande importância de investigar os impactos que as OCs podem causar nas populações que residem no entorno das unidades de conservação 
(UCs). Tendo em vista o cenário de mudanças climáticas e o aumento nas ocorrências das OCs e sua relação com o desconforto térmico humano, este estudo busca investigar o seu impacto na população (dentre elas indígenas) que vive na região da Floresta Nacional dos Tapajós (FNT), localizada no estado do Pará, região Norte do Brasil.

\section{Material e Métodos}

\section{Área de estudo}

A FNT é uma UC federal localizada no bioma da Amazônia, criada em 19 de fevereiro de 1974, cuja localização está contida na Figura 1. A FNT possui uma área de 527.319ha, abrangendo os municípios de Aveiro, Belterra, Placas e Rurópolis, no oeste do estado do Pará. Essa UC busca um uso sustentável dos seus recursos naturais, o que possibilitou o manejo florestal comunitário em uma área reservada para essa finalidade. Atualmente residem na FNT cerca de
1.050 famílias, aproximadamente 4 mil moradores (lotes com assentados do Incra, proprietários e posseiros), em 23 comunidades e três aldeias indígenas da etnia Munduruku. A FNT apresenta ainda cerca de 160 quilômetros de praias de água doce e uma grande diversidade de paisagens naturais: rios, lagos, alagados, terra firme, morros, planaltos, floresta, campos, açaizais; o que faz com o que o turismo seja uma importante atividade nesta região (ICMBio, 2020).

Segundo Fisch (1998), a região de estudo apresenta altos valores de radiação solar incidente, sendo os maiores nos meses de setembro/outubro $e$ os menores nos meses de dezembro a fevereiro. A precipitação é um importante elemento climático, pois induz variáveis como a temperatura $e$ umidade; na região de estudo o período de maior precipitação é observado no período de dezembro a maio. Contudo, é registrado uma pequena variação da temperatura do ar ao longo do ano, onde o máximo é observado em novembro $\left(26,5^{\circ} \mathrm{C}\right)$ e o mínimo em março $\left(25,4^{\circ} \mathrm{C}\right)$ (Fisch et al., 1998).
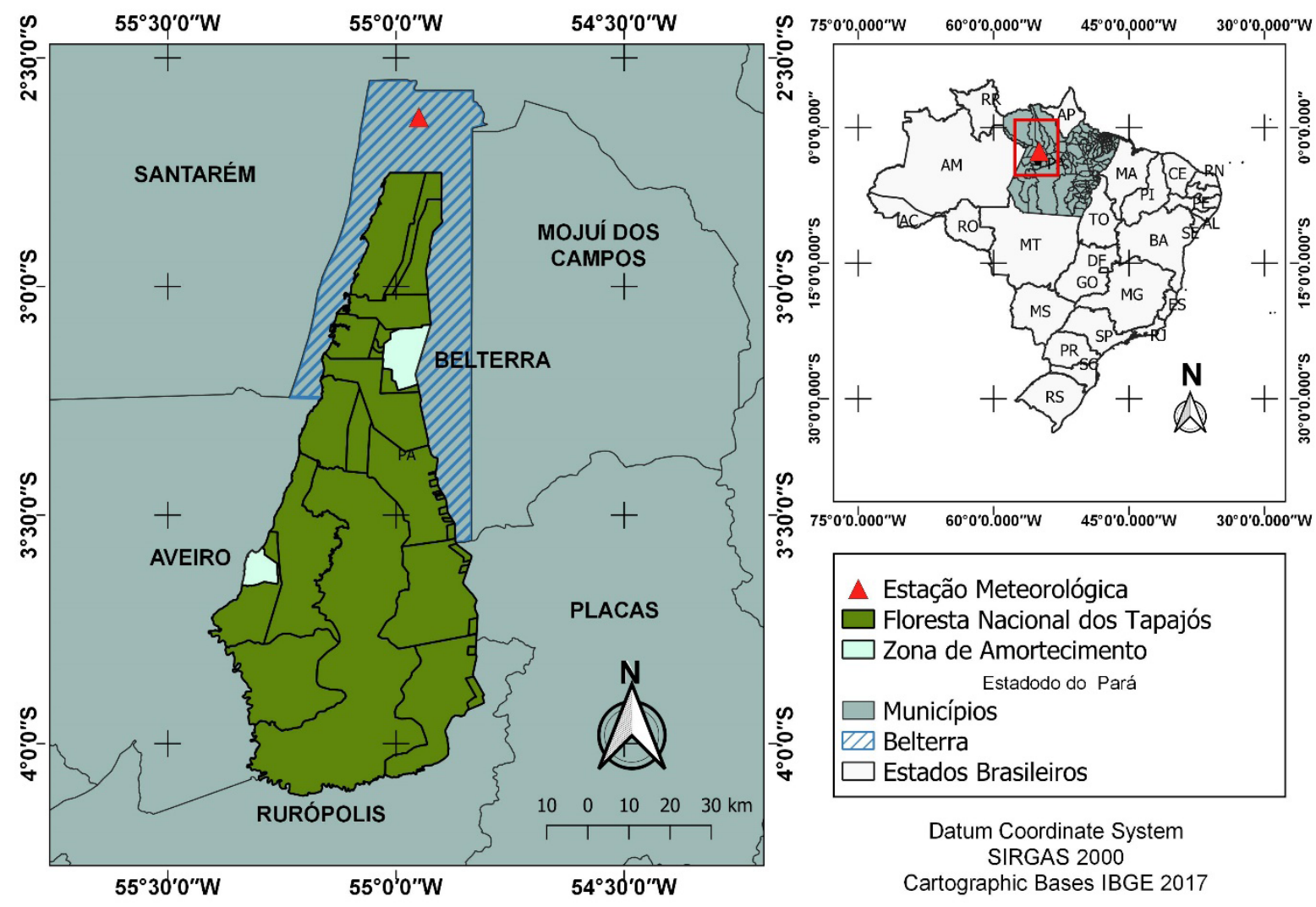

Figura 1 - Localização da Floresta Nacional dos Tapajós (hachurado em verde), a oeste do estado do Pará, na região Norte do Brasil. 


\section{Dados}

Foram utilizados dados diários de umidade relativa do ar, temperatura média e máxima do ar registrados pela estação meteorológica convencional número 82246 situada na cidade de Belterra (2॰37'48'S e 5457'W), disponibilizados pelo Instituto Nacional de Meteorologia (INMET), a partir do Banco de Dados Meteorológicos para Ensino e Pesquisa (BDMEP). No presente estudo considerou-se o período de 1971 a 2019, a utilização dos dados da estação de Belterra localizada no entorno da FNT é baseada na necessidade de se ter no mínimo 30 anos consecutivos para identificar as OCs, conforme será mostrado posteriormente.

\section{Consistência e preenchimento dos dados}

Erros na medição, armazenamento e leitura de registros meteorológicos podem ocorrer, mesmo em registros fornecidos por banco de dados oficiais como o INMET, que já contam com sistema de controle dos dados. Portanto, o procedimento de consistência dos dados visa detectar erros ocorridos no processo de registro, formatação, transmissão $e$ arquivamento dos dados meteorológicos, para que esses dados possam ser utilizados da melhor forma possivel (WMO, 2008). Este procedimento foi realizado utilizando a metodologia da variação interquartil (n) baseada nos quartis estatísticos, proposta por Sugahara et al. (2012) que é dada a partir da equação 1.

$$
\mathrm{n}=\mathrm{Q} 3 \pm(3 * \mathrm{~d})
$$

onde $\mathrm{n}$ são os intervalos positivos e negativos, em que todos os dados acima e abaixo desse intervalo, respectivamente, são removidos; Q3 é o quartil $75 \%$ e do intervalo interquartil.

Foram observadas falhas de $18,7 \%$ na umidade relativa do ar (UR), $17,1 \%$ temperatura média do ar (TM) e de $16,6 \%$ na temperatura máxima do ar (TMáx), que foram preenchidos utilizando imputação múltipla via Multivariate
Imputation by Chained Equations (MICE), metodologia estatística baseada no amostrador de Gibbs que possui diferentes métodos de imputação (Azur et al., 2011). Na presente pesquisa, foi adotado o método da Média Preditiva Correspondente (MPC), que é uma alternativa do modelo de regressão linear, que determina um valor imputado calculado pelo modelo de regressão mais próximo do valor observado baseado na série de dados em interesse (Li et al., 1991). A escolha da MPC é baseada no desempenho satisfatório que este método apresentou em dados meteorológicos apresentado por Alves \& Gomes (2020), sendo obtida de acordo com a equação 2 .

$$
\hat{\mathrm{Y}}^{\mathrm{obs}}=\left\{\mathrm{Y}_{\mathrm{i}}^{\mathrm{obs}}=\mathrm{X}^{\prime} \mathrm{i} \beta^{*} ;_{\mathrm{i}} \in \text { obs }(\mathrm{Y})\right\}
$$

sendo $\mathrm{X}$ a variável meteorológica com registros faltante, $\hat{\mathrm{Y}}^{\text {obs }}$ o grupo dos valores observados $e$ $\mathrm{Y}_{\mathrm{i}}^{\mathrm{obs}}=\mathrm{X}^{\prime} \mathrm{i} \beta^{*}$ que retorna $\mathrm{Y}_{\mathrm{i}}^{\mathrm{obs}}$ como observação mais próxima.

\section{Mensuração empírica do conforto térmico humano}

O conforto térmico humano foi estimado a partir do Índice de Desconforto de Thom (ID), uma metodologia empírica que a partir de registros de TM e UR estima a sensação térmica do ser humano (THOM, 1959). A escolha do ID foi baseada na praticidade de cálculo e pelo desempenho satisfatório que o mesmo apresentou na estimativa do conforto térmico em Santarém, cidade próxima à região em estudo (Mandú et al., 2020b). O ID é calculado a partir da equação 3:

$$
\mathrm{ID}=\mathrm{TM}-0,55 *(1-0,01 * \mathrm{UR}) *(\mathrm{TM}-14,5)
$$

onde ID é o índice de desconforto $\left({ }^{\circ} \mathrm{C}\right) ; \mathrm{TM}$ a temperatura média do ar $\left({ }^{\circ} \mathrm{C}\right)$, e UR a umidade relativa do ar (\%).

A adaptação das faixas do ID para a região equatorial é apresentada por Santos et al. (2012) estão contidas na Tabela 1.

Tabela 1 - Faixas do ID para regiões tropicais.

\begin{tabular}{|c|c|}
\hline Faixas & Valor do ID $\left({ }^{\circ} \mathbf{C}\right)$ \\
\hline Confortável & ID $<24$ \\
\hline Parcialmente confortável & $24 \leq$ ID $<26$ \\
\hline Desconfortável & $26 \leq$ ID $<28$ \\
\hline Muito desconfortável & ID $\geq 28$ \\
\hline
\end{tabular}




\section{Identificação dos episódios de ondas de calor}

A identificação das OCs foi realizada utilizando o índice CTX90pct, que considera uma OC quando por 3 dias consecutivos ou mais a TMáx fica acima do percentil 90 climatológico, onde é calculado um percentil 90 para cada dia do ano, centrado no dia em questão em uma média móvel de 15 dias, calculando valor diferente de percentil 90 para cada dia do ano, reduzindo assim os efeitos de sazonalidade (Perkins et al., 2012; Geirinhas et al., 2018). Portanto, para um dado dia d, o limiar é o percentil 90 do conjunto de dados por:obtido a partir da equação 4 .

$$
A_{d}=\bigcup_{y=1971 \mathrm{i}=\mathrm{d}-7}^{\mathrm{d}+8} \bigcup_{m} \operatorname{máx}, \mathrm{i}
$$

onde $U$ é a união de conjuntos; Tmáx, é a temperatura máxima do ar diária do dia i no ano y.

\section{Métodos}

As análises foram realizadas a partir de comparações do ID durante episódios com e sem OC em escala anual, mensal e diária. Além disso, foram avaliados parâmetros de estatística descritiva, que possibilitaram avaliar as características da posição e dispersão das OCs, com o objetivo de avaliar o impacto das OCs no conforto térmico humano. Avaliou-se também a distribuição de probabilidade do ID na presença $e$ ausência das OCs a fim de verificar se a ocorrência do fenômeno altera a distribuição do ID. O teste $\mathrm{t}$ de Student serviu para comparar as médias do ID durante eventos com e sem OC, para verificar se as OCs alteram o comportamento médio do conforto térmico humano na região da FNT. O teste $\mathrm{t}$ de Student possui as seguintes hipóteses: Nula $\left(\mathrm{H}_{0}\right)$ os dados fornecem evidências de que as médias são iguais; Alternativa $\left(\mathrm{H}_{1}\right)$ não há argumentos suficientes para aceitar $\mathrm{HO}$, ou seja, as médias apresentam diferenças considerando o nível de significância de 5\% (Wilks, 2011).

\section{Resultados e Discussões}

Na Tabela 2 são apresentadas as estatísticas descritivas da temperatura média e umidade relativa do ar na ausência e presença dos episódios de ondas de calor. Onde é possível observar que durante os eventos a temperatura apresenta maior média e menor variabilidade conforme notado pelo desvio padrão e variância, também é notado que a amplitude é inferior. Para a umidade relativa do ar é vista uma redução na média e variabilidade, indicando que a ocorrência de eventos de ondas de calor ocasiona no aumento da temperatura $e$ redução na umidade relativadoar, concordando com FISCHER \& SCHÄR, 2009.

Tabela 2 - Estatística descritiva da temperatura média e umidade relativa do ar com a presença e ausência dos eventos de ondas de calor.

\begin{tabular}{|c|c|c|c|c|}
\hline \multirow{2}{*}{ Estatísticas } & \multicolumn{2}{|c|}{ Sem ondas de calor } & \multicolumn{2}{c|}{ Com ondas de calor } \\
\cline { 2 - 5 } & $\begin{array}{c}\text { Temperatura média do ar } \\
\left({ }^{\circ} \mathbf{C}\right)\end{array}$ & $\begin{array}{c}\text { Umidade relativa do ar } \\
(\mathbf{\%})\end{array}$ & $\begin{array}{c}\text { Temperatura média do ar } \\
\left({ }^{\circ} \mathbf{C}\right)\end{array}$ & $\begin{array}{c}\text { Umidade relativa do ar } \\
(\mathbf{\%})\end{array}$ \\
\hline Máximo & 30,24 & 99,50 & 31,16 & 94,5 \\
\hline Média & 25,47 & 87,69 & 27,32 & 82,5 \\
\hline Desvio-padrão & 1,19 & 5,39 & 0,89 & 5,21 \\
\hline Variância & 1,42 & 29,1 & 0,79 & 27,2 \\
\hline Amplitude & 12,0 & 36,5 & 6,38 & 32,5 \\
\hline Mínimo & 18,2 & 63,00 & 24,78 & 62,0 \\
\hline
\end{tabular}

Na Tabela 3 é apresentada a estatística descritiva das condições de conforto térmico humano com e sem a presença dos eventos de OC. É possível observar que o ID na ausência das OCs é de $24,7^{\circ} \mathrm{C}$ (parcialmente confortável), e as temperaturas médias apresentaram-se na ordem de $26,1^{\circ} \mathrm{C}$ durante a ocorrência dos episódios de OC. Destaca-se também os valores de desviopadrão, variância e amplitude que são menores durante as OCs, indicando que durante esses eventos o ID apresenta menor variabilidade. 
Tabela 3 - Estatística descritiva do ID com a presença e ausência dos eventos de ondas de calor.

\begin{tabular}{|c|c|c|}
\hline Estatísticas & Sem ondas de calor & Com ondas de calor \\
\hline Máximo $\left({ }^{\circ} \mathrm{C}\right)$ & 28,9 & 29,3 \\
\hline Média $\left({ }^{\circ} \mathrm{C}\right)$ & 24,7 & 26,1 \\
\hline Desvio-padrão $\left({ }^{\circ} \mathrm{C}\right)$ & 0,93 & 0,59 \\
\hline Variância $\left({ }^{\circ} \mathrm{C}\right)$ & 0,87 & 0,35 \\
\hline Amplitude $\left({ }^{\circ} \mathrm{C}\right)$ & 10,8 & 5,12 \\
\hline Mínimo & 18,1 & 24,2 \\
\hline
\end{tabular}

Nota-se, também, elevação no desconforto térmico sentido pela população na região da FNT (Figura 2), assim como a baixa ocorrência de OC até o ano de 1995, sem eventos de OC nos nove primeiros anos, que pode estar relacionada com processos de alterações no microclima (escala local) e também as mudanças climáticas de escala global (Luo et al., 2020). Além disso, nota-se que os anos de 1997-1998 e 2015 são caracterizados por altos valores de ID durante eventos de OC, anos que também corresponderam a períodos de forte estiagem na região, de acordo com JiménezMunõz et al. (2016). Um aumento no ID durante os anos de 1997-1998 na ausência de OC também foi notado, ao contrário do que foi observado em 2015 , em que o impacto no desconforto foi maior durante eventos de OC.

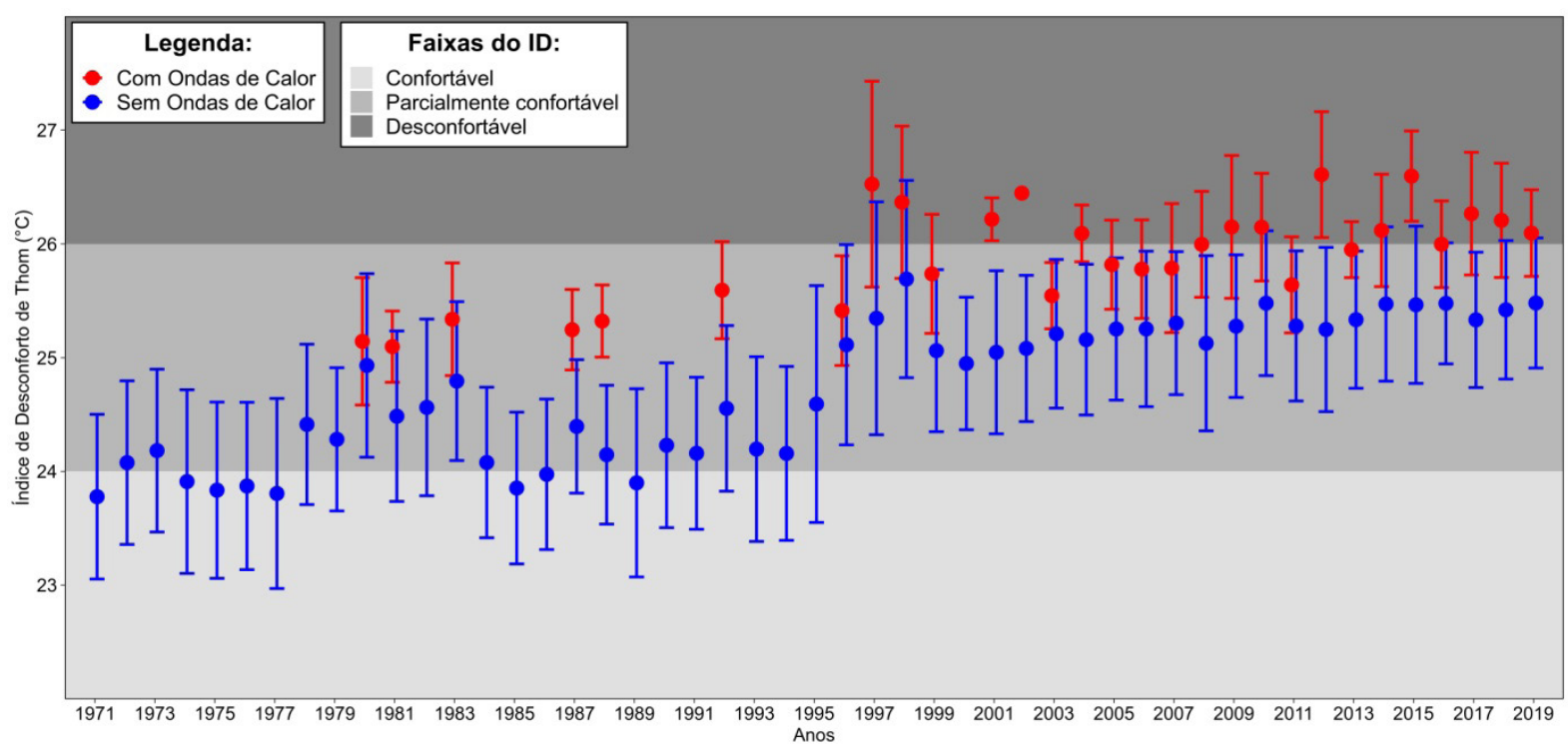

Figura 2 - Comportamento anual do ID com (vermelho) e sem (azul) ondas de calor. Bolinhas representam a média; e as barras, o desvio-padrão.

Com relação ao comportamento sazonal (Figura 3), as médias do ID foram maiores durante os eventos de OC (linha vermelha), com um máximo em novembro $\left(26,7^{\circ} \mathrm{C}\right)$ e mínimo de $25,6^{\circ} \mathrm{C}$ em fevereiro, enquanto que o ID na ausência de eventos de OC foi maior em outubro $\left(25,4^{\circ} \mathrm{C}\right)$ e menor em julho $\left(24,3^{\circ} \mathrm{C}\right)$. Segundo
Parsons (2014), altos níveis de radiação, somados a altas temperatura e umidade contribuem para a elevação do desconforto térmico humano, características observadas na região de estudo. Mandú et al. (2020a) mostraram que no período de agosto a dezembro há um maior desconforto térmico por calor na região, por se tratar da 
estação menos chuvosa; o que evidencia o importante papel da precipitação no resfriamento da atmosfera, que contribui para aumentar a sensação de conforto na estação chuvosa.

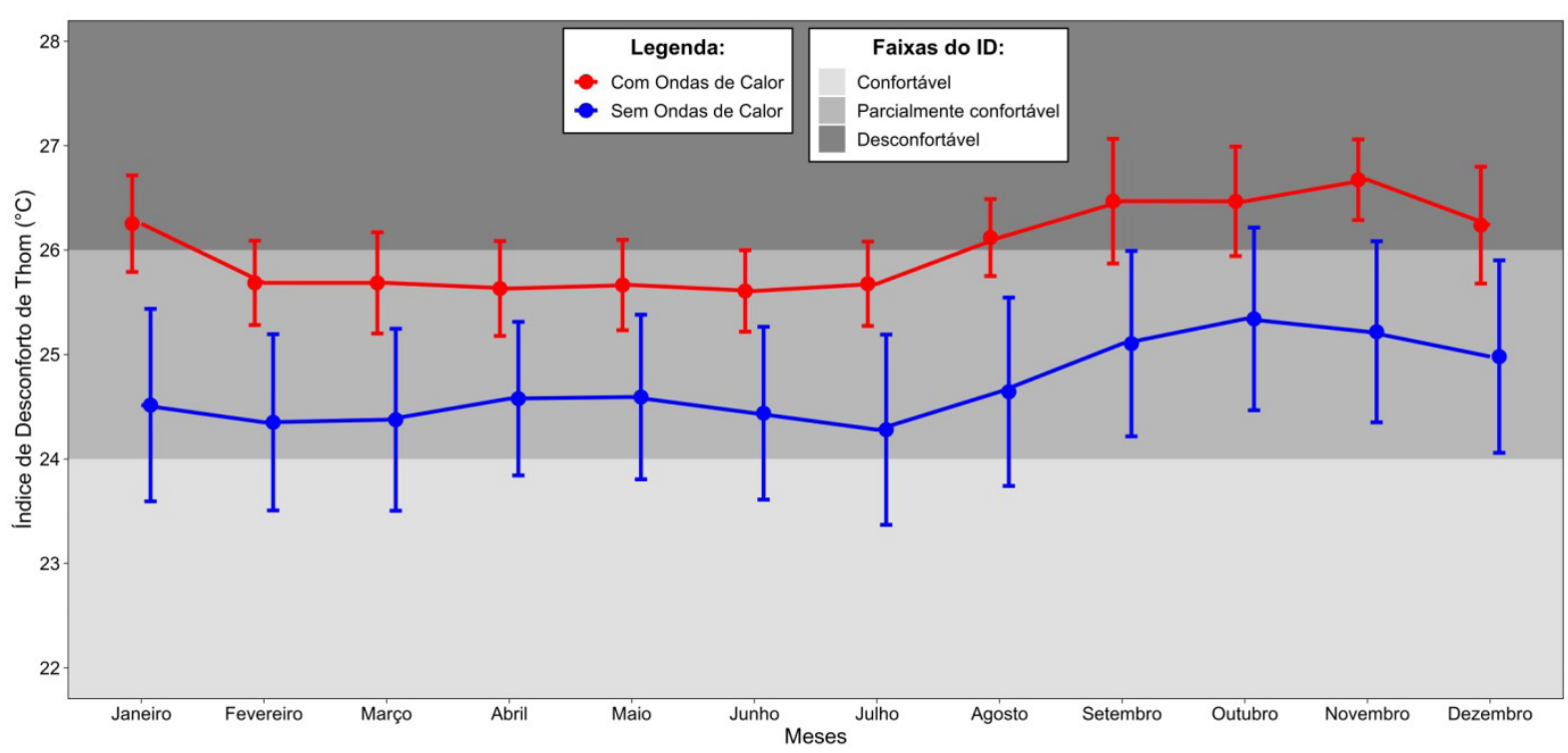

Figura 3 - Comportamento mensal do ID com ondas de calor (vermelho) e sem (azul). Bolinhas representam a média; e as barras, o desvio-padrão.

Com relação a escala diária (Figura 4), na ausência de eventos de OC foi observado um comportamento mais homogêneo, que apresenta dois períodos bem definidos: entre 10 de dezembro e 7 de julho, que correspondem a estação chuvosa e a estação de transição, respectivamente; a segunda, entre 8 julho e 9 de dezembro, em que é observada a estiagem na região e também onde os maiores valores de ID são observados. Para o ID durante episódios de OC (linha vermelha) é notada maior variabilidade durante o ano inteiro. Somente 24 dias não apresentaram presença do fenômeno durante o período analisado (19712019), equivalente a $6,6 \%$ do total de dias, o que mostra que a região é suscetível a ocorrência de OC durante maior parte do ano.

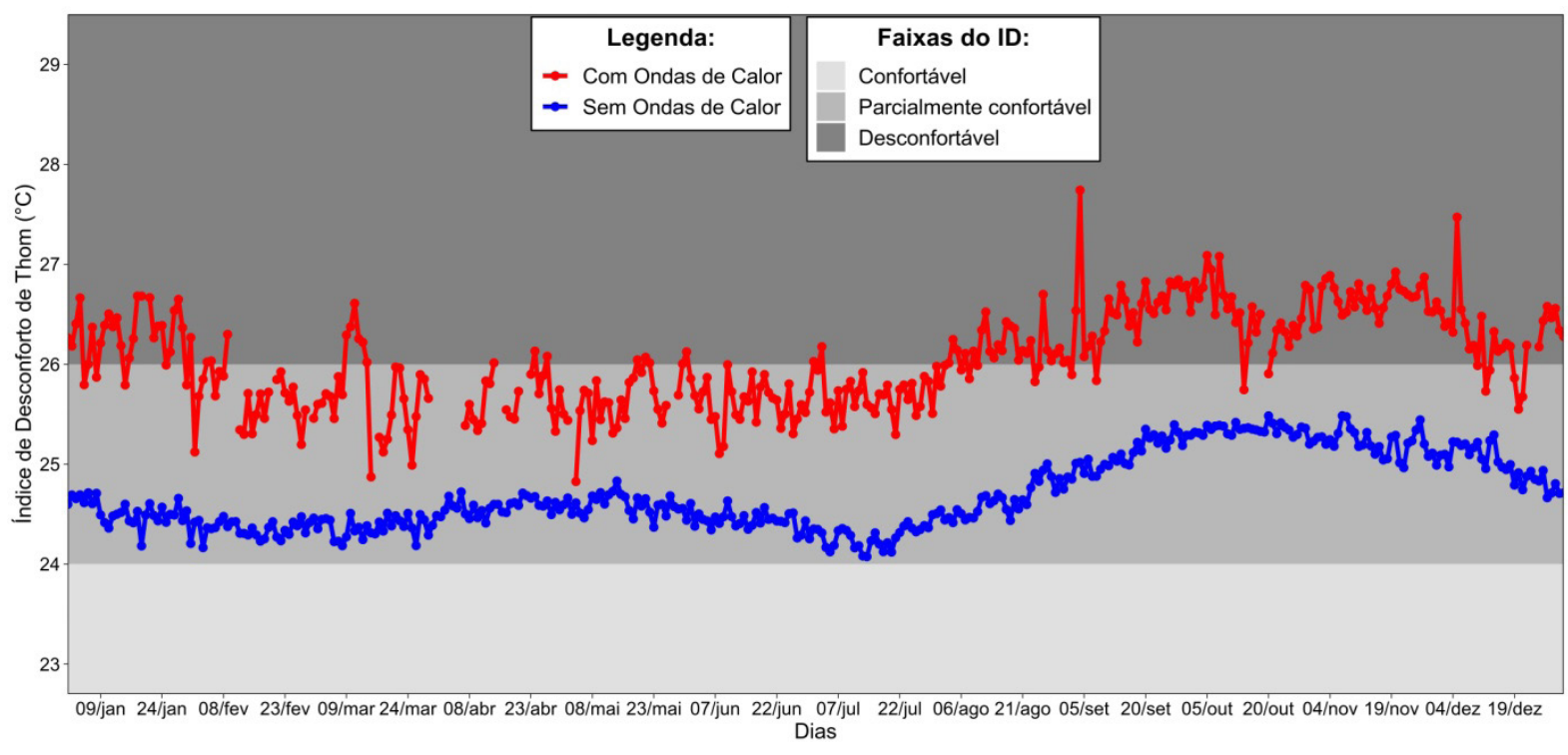

Figura 4 - Comportamento diário do ID com OC (linha vermelha) e sem OC (linha azul). 
Na Figura 5 é apresentada a quantidade dias (em porcentagem) para as escalas do ID (Tabela 1). Para os dias em que não houveram ocorrências de OC, a maioria dos dias (70,3\%) correspondem a períodos parcialmente confortáveis, $22,1 \%$ correspondem a períodos confortáveis e apenas $7,6 \%$ para períodos desconfortáveis. Durante os eventos de OC não foram observados dias confortáveis, sendo $54,1 \%$ dos dias caracterizados como desconfortável, $43,8 \%$ como confortáveis e apenas $0,1 \%$ dos dias foram muito desconfortáveis.

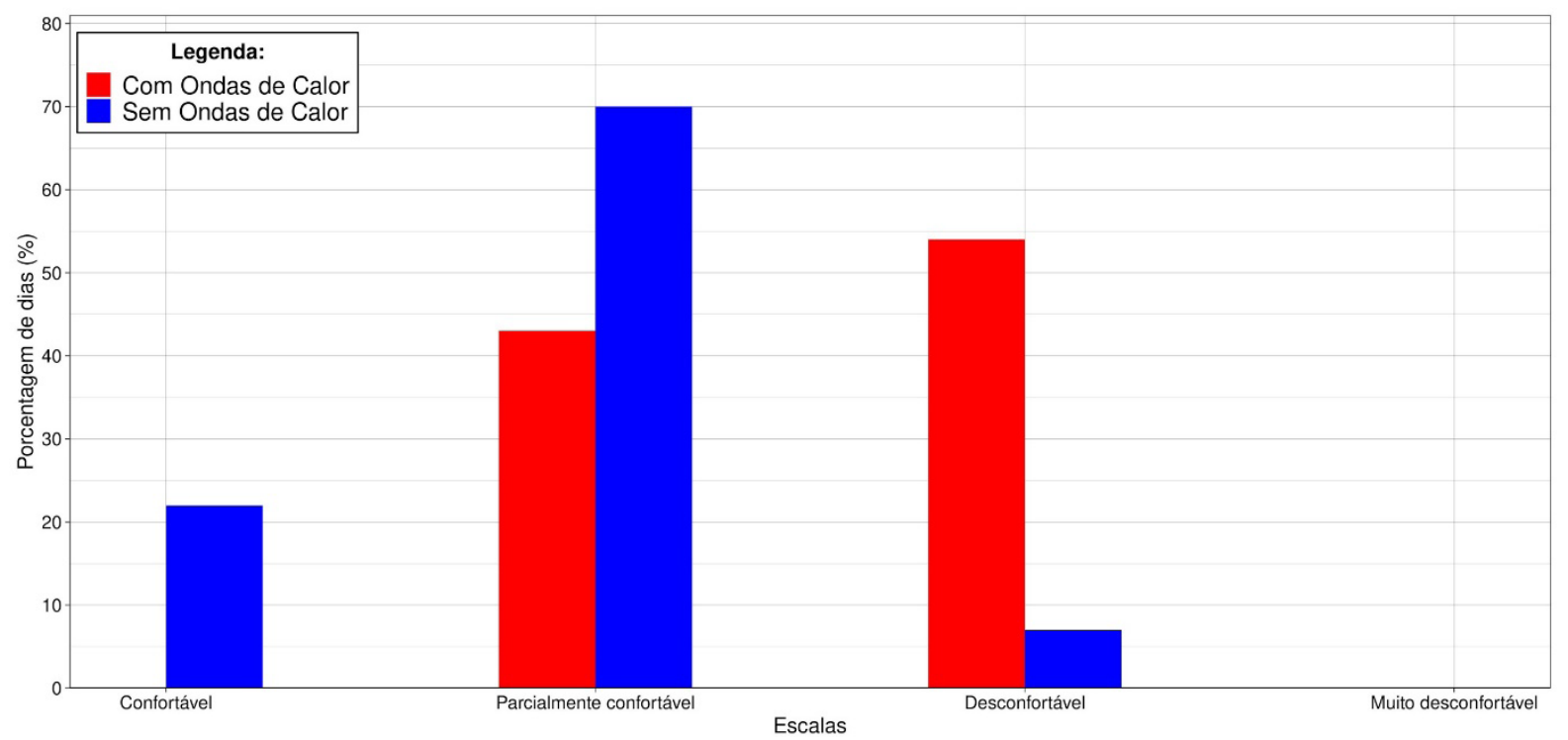

Figura 5 - Porcentagem de dias por faixa do ID. As barras em azul correspondem a dias sem ocorrências de OC, $e$ as barras em vermelho correspondem a dias com ocorrência de OC.

A Figura 6 apresenta a distribuição de probabilidade do ID. Na ausência de OC é observado que o ID possui comportamento com maior uniformidade entre $22^{\circ} \mathrm{C}$ e $27^{\circ} \mathrm{C}$, que se assemelha a uma distribuição normal, diferente do observado no ID durante os eventos de calor onde a curva é mais achatada, com valores entre $24^{\circ} \mathrm{C}$ e $28^{\circ} \mathrm{C}$, mostrando que a ocorrência desse evento altera o comportamento do ID na FNT; indicando direta influência no desconforto térmico dos habitantes dessa região.

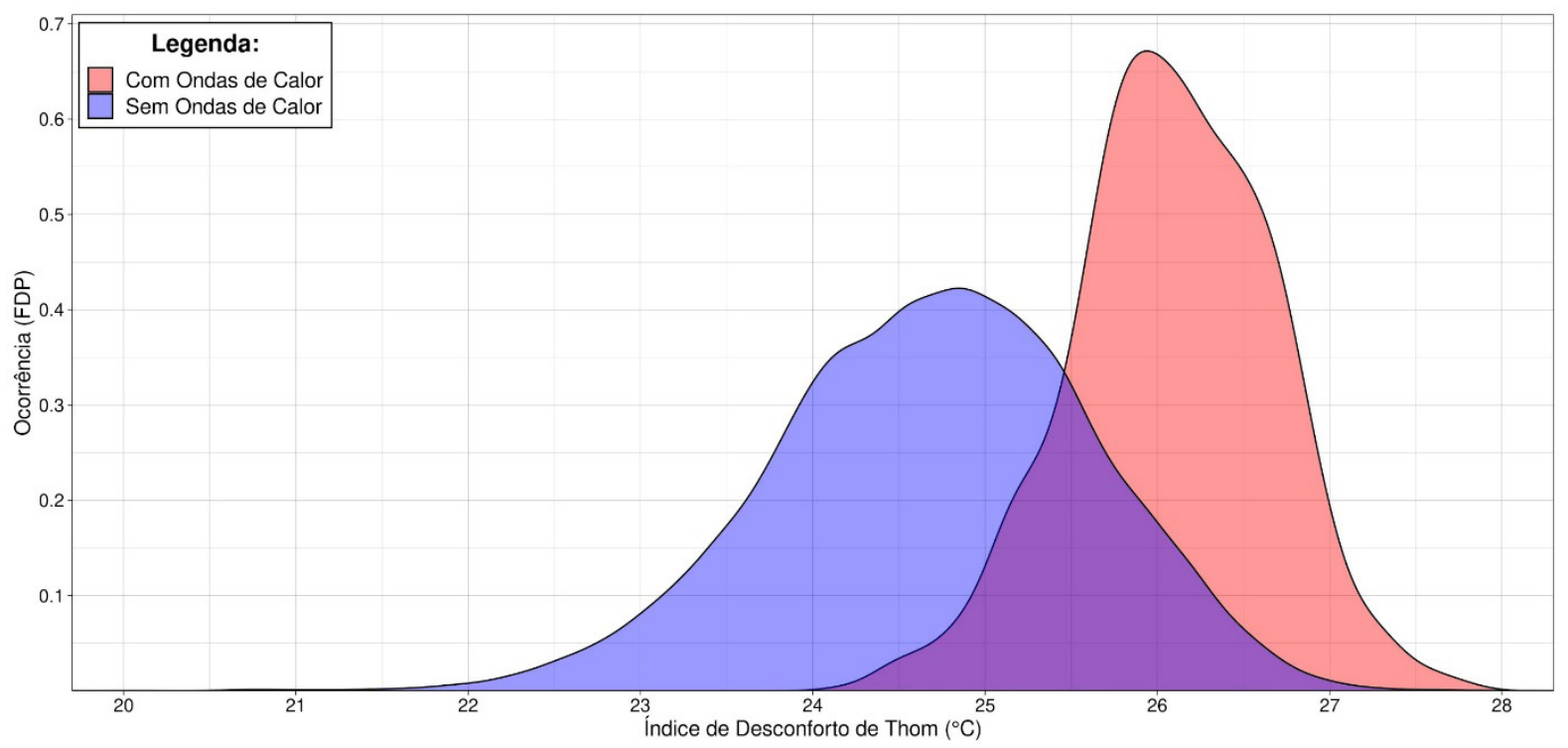

Figura 6 - Distribuição do ID na ausência de ondas de calor (em azul) e na presença de OC (em vermelho). 
Na Tabela 4 é apresentado o resultado do teste $\mathrm{t}$ de Student. O teste $\mathrm{t}$ indica que as médias do ID são estaticamente significativas ao nível de confiança de $5 \%$ tanto na presença como na ausência de eventos de OC. Os resultados apresentados indicam que as OCs são responsáveis por alterar o conforto térmico humano na região da FNT e podem influenciar significativamente as condições térmicas experimentadas pelas pessoas que habitam nesta região.

Tabela 4 - Teste t de Student entre o ID durante eventos de OC e do ID na ausência desses eventos.

\begin{tabular}{|c|c|}
\hline Estatística do teste & Valor-p \\
\hline 69,019 & $<0,001$ \\
\hline
\end{tabular}

Os resultados encontrados neste estudo concordam com o que foi visto em Bitencourt et al. (2020), onde os autores mostram um aumento das OCs em todas as regiões do Brasil. Por se tratar de uma região pouco urbanizada, normalmente não é esperado os impactos das temperaturas extremas, entretanto, neste estudo foi possível observar que esse comportamento não está restrito somente a grandes centros urbanos. Dito isto, salienta-se a necessidade de estudos posteriores voltados para a avaliação de como esse aumento pode afetar a saúde e bem-estar da população local, uma vez que como mostrado por Opitz-Stapleton et al. (2016), afetam diretamente diversas atividades realizadas na FNT.

Apesar da limitação de não haver dados suficientes de registros meteorológicos para identificar as OCs dentro da FNT, a distância da estação de Belterra a região da FNT é pequena $e$, portanto, serviu para cumprir o objetivo de avaliar preliminarmente o impacto que as OCs podem trazer para o local. Outra limitação se refere ao índice de desconforto adotado, por ser uma metodologia empírica, não foi considerado a percepção pessoal dos moradores da região, porém, fornece informações gerais de como seria as condições térmicas sentidas pelos habitantes.

\section{Conclusões}

O estudo mostrou a influência das OCs no desconforto térmico humano na região da Floresta Nacional do Tapajós. A partir de uma análise descritiva foi possível observar que os dias desconfortáveis são cerca de $40 \%$ superiores durante os episódios de OC, influenciando no comportamento anual, mensal e diário deste desconforto. O desconforto é superior em anos de fortes eventos de seca na região e durante a estação menos chuvosa, com expressiva alteração na distribuição nas faixas do ID.

Salienta-se a importância do presente estudo, diante do impacto negativo que o desconforto térmico pode causar na saúde e no bem-estar da população, que instiga a tomada de medidas públicas que visem reduzir os impactos negativos deste excessivo desconforto térmico, como a criação de sistemas de alerta para as OCs e de informações na prevenção durante os eventos, assim como pesquisas que busquem avaliar como esse desconforto afeta na saúde e no bem-estar dos moradores da Floresta Nacional do Tapajós.

\section{Referências}

Alves LER \& Gomes HB. Validação da Imputação Múltipla via Predictive Mean Matching para Preenchimento de Falhas nos Dados Pluviométricos da Bacia do Médio São Francisco. Anuário do Instituto de Geociências, 43(1): 199-206, 2020.

Artaxo P. Mudanças climáticas e o Brasil. Revista USP, 103: 8-12, 2014.

Azur MJ, Stuart EA, Frangakis C \& Leaf PJ. Multiple imputation by chained equations: what is it and how does it work? International journal of methods in psychiatric research, 20(1): 40-49, 2011.

Silva PL de A. Biodiversidade e mudanças climáticas no Brasil: levantamento e sistematização de referências. WWF Brasil (Relatório). Brasília, 2018.

Bitencourt DP, Fuentes MV, Maia PA \& Amorim FT. Frequência, Duração, Abrangência Espacial $e$ Intensidade das Ondas de Calor no Brasil. Revista Brasileira de Meteorologia, 31(4): 506-517, 2016.

Bitencourt DP, Fuentes MV, Franke AE, Silveira RB \& Alves MP. The climatology of cold and heat waves in Brazil from 1961 to 2016. International Journal of Climatology, 40(4): 2464-2478, 2020. 
Fischer EM \& Schär C. Future changes in daily summer temperature variability: driving processes and role for temperature extremes. Climate Dynamics, 33(7-8): 917, 2009.

Fisch G, Marengo JA \& Nobre CA. The climate of Amazonia - a review. Acta Amazônica, (28)2: 101-126, 1998.

Frich $\mathrm{P}$ et al. Observed coherent changes in climatic extremes during the second half of the twentieth century. Climate research, 19: 193-212, 2002.

Geirinhas JL, Trigo RM, Libonati R, Coelho CA \& Palmeira AC. Climatic and synoptic characterization of heat waves in Brazil. International Journal of Climatology, 38(4): 1760-1776, 2017.

Geirinhas JL, Trigo RM, Libonati R \& Peres LDF. Caracterização Climática de Ondas de Calor no Brasil. Anuário do Instituto de Geociências, 41(3): 333-350, 2019.

Geirinhas JL et al. Heat-related mortality at the beginning of the twenty-first century in Rio de Janeiro, Brazil. International journal of biometeorology, 1-14, 2020.

ICMBio - Instituto Chico Mendes de Conservação da Biodiversidade. Disponivel em: https://www.icmbio. gov.br/flonatapajos/. Acesso em: 10/07/2020.

Jacondino WD, Nascimento ALDS, Nunes AB \& Conrado H. Análise sinótica do mês de abril de 2018 na região Sul do Brasil: Episódio de calor extremo. Revista Brasileira de Climatologia, (25): 182-203, 2019.

Jenerette GD et al. Regional relationships between surface temperature, vegetation, and human settlement in a rapidly urbanizing ecosystem. Landscape ecology, 3(22): 353-365, 2007.

Jiménez-Muñoz JC et al. Record-breaking warming and extreme drought in the Amazon rainforest during the course of El Niño 2015-2016. Scientific reports, (6): 33130, 2016.

Kong Q, Guerreiro SB, Blenkinsop S, Li XF \& Fowler HJ. Increases in summertime concurrent drought and heatwave in Eastern China. Weather and Climate Extremes, 28(1): 100242, 2020.

Laaidi K et al. The impact of heat islands on mortality in Paris during the August 2003 heat wave. Environmental health perspectives, 2(120): 254-259, 2012.

Li KH, Raghunathan TE \& Rubin DB. Large-sample significance levels from multiply imputed data using moment-based statistics and an $\mathrm{F}$ reference distribution. Journal of the American Statistical Association, 86(416): 1065-1073, 1991.

Lima NGB, Galvani E, Falcão RM \& Cunha-Lignon M. Air temperature and canopy cover of impacted and conserved mangrove ecosystems: a study of a subtropical estuary in Brazil. Journal of Coastal Research, (65): 1152-1157, 2013.

Luo M, Ning G, Xu F, Wang S, Liu Z \& Yang Y. Observed heatwave changes in arid northwest China: Physical mechanism and long-term trend. Atmospheric Research, 1(1): 105009, 2020.

Mandú TB, Gomes ACDS, Costa GB \& Neves TTDAT. Avaliação de tendência nas ondas de calor registradas em Manaus/AM, Brasil. Revista Brasileira de Climatologia, 27(1): 405-425, 2020a.

Mandú TB, Gomes ACDS \& Coutinho MDL. Caracterização do conforto térmico da cidade de Santarém/PA. Revista Geonorte, 11(37): 279-291, $2020 b$.

Marengo JA. O futuro clima do Brasil. Revista USP, 103: 25-32, 2014.

Marengo JA \& Valverde MC. Caracterização do clima no Século XX e Cenário de Mudanças de clima para o Brasil no Século XXI usando os modelos do IPCC-AR4. Revista Multiciência, 8: 5-28, 2007.

McGeehin MA \& Mirabelli M. The potential impacts of climate variability and change on temperaturerelated morbidity and mortality in the United States. Environmental health perspectives, 2(109): 185-189, 2001.

Mitchell $\mathrm{D}$ et al. Attributing human mortality during extreme heat waves to anthropogenic climate change. Environmental Research Letters (11): 074006, 2016.

Nick LM \& Nedel AS. Análise do Conforto Térmico Humano ao Longo do Verão na Cidade de Pelotas/RS e a Relação com Condições Meteorológicas Extremas. Anuario do Instituto de Geociencias, 2(41): 211-222, 2018.

Opitz-Stapleton $\mathrm{S}$ et al. Heat index trends and climate change implications for occupational heat exposure in Da Nang, Vietnam. Climate Services (2): 41-51, 2016.

Parsons K. 2014. Human thermal environments: the effects of hot, moderate, and cold environments on human health, comfort, and performance. $1 \mathrm{ed}$. CRC press. 545p.

Perkins SE, Alexander LV \& Nairn JR. Increasing frequency, intensity and duration of observed global heatwaves and warm spells. Geophysical Research Letters, 39(20): 1-5, 2012.

Reis NCS, Boiaski NT \& Ferraz SET. Characterization and Spatial Coverage of Heat Waves in Subtropical Brazil. Atmosphere, 10(5): 1-15, 2019.

Rusticucci M \& Barrucand M. Climatología de temperaturas extremas en la Argentina. Consistencia de datos. Relación entre la temperatura media estacional 
y la ocurrencia de días extremos. Meteorologica, (26): 69-84, 2002.

Santos J, Silva V, Lima E, Araújo L \& Costa A. Campo Térmico Urbano e a sua Relação com o Uso e Cobertura do Solo em Cidade Tropical Úmida. Revista Brasileira de Geografia Física, 5(3): 540-557, 2012.

Shiva J, Chandler DG \& Kunkel KE. Localized changes in heat wave properties across the United States. Earth's Future, 7(3): 300-319, 2019.

Sugahara S, Rocha RP, Ynoue RY \& Silveira RB. Homogeneity assessment of a station climate series (1933-2005) in the Metropolitan Area of São Paulo: instruments change and urbanization effects. Theoretical and applied climatology, 107(3-4): 361-374, 2012.
Stott PA et al. Attribution of weather and climaterelated events. Climate science for serving society, 1(1): 307-337, 2013.

Thom EC. The discomfort index. Weatherwise, (12)2: 57-61, 1959.

Wilks DS. 2011. Statistical methods in the atmospheric sciences. 3 ed. Academic press. 661p.

WMO. World Meteorological Organization. Guide to Meteorological Instruments and Methods of Observation. Note $n^{\circ} 8$, Secretariat of the WMO, Geneva, 2008.

Biodiversidade Brasileira - BioBrasil.

Edição Temática: Análise de Componentes do Sistema Climático e a Biodiversidade no Brasil

$$
\text { n. } 4,2021
$$

http://www.icmbio.gov.br/revistaeletronica/index.php/BioBR

Biodiversidade Brasileira é uma publicação eletrônica científica do Instituto Chico Mendes de

Conservação da Biodiversidade (ICMBio) que tem como objetivo fomentar a discussão e a disseminação de experiências em conservação e manejo, com foco em unidades de conservação $e$ espécies ameaçadas.

ISSN: 2236-2886 